\title{
Réflexions sur la conférence de Jean-Hubert Lavie
}

Comments on J.H. Lavie's article

\section{Christian Fonnet}

\section{CpenEdition}

\section{Journals}

Édition électronique

URL : https://journals.openedition.org/rhcf/88

DOI : 10.4000/rhcf.88

Éditeur

Rails \& histoire

Édition imprimée

Date de publication : 1 décembre 2007

Pagination : 60-63

ISSN : 0996-9403

Référence électronique

Christian Fonnet, "Réflexions sur la conférence de Jean-Hubert Lavie », Revue d'histoire des chemins de fer [En ligne], 36-37 | 2007, mis en ligne le 10 mai 2011, consulté le 22 avril 2022. URL : http:// journals.openedition.org/rhcf/88; DOI : https://doi.org/10.4000/rhcf.88 


\title{
Réflexions sur la conférence de Jean-Hubert Lavie
}

\author{
Christian Fonnet \\ Service photographique des éditions La Vie du rail
}

Il existe assez peu de photographies de cheminots sur leur lieu de travail car les prises de vue étaient interdites, les chemins de fer étant considérés comme des installations à caractère stratégique. On trouve surtout des photos de cheminots en groupe, dans la gare, ou sur leur locomotive mais très rarement des vues générales d'installations, de signalisation ou de postes d'aiguillage.

Les photos officielles existent, souvent classées par grades des agents, notamment dans les ateliers. Quelques cheminots amateurs ont tenté d'imiter la photo officielle, tel Albert Henry à l'Est, pour la gloire de la locomotive à vapeur, certes, mais plus peut-être pour la Compagnie de chemin de fer. Lucien-Maurice Vilain fit de même pour son cher PO. Ces photographes ont rarement pris de clichés de voyageurs. Albert Henry fit dessiner les couvre-roues portant les noms germaniques des locomotives (démontés pendant la Grande Guerre pour des raisons patriotiques) qu'il replaçait avant de prendre sa photo. Pour un tel puriste, la locomotive formait un tout et pas un détail ne devait manquer. Notons que ses photos s'arrêtent à la nationalisation. L.-M. Vilain a pris aussi pas mal de plaques à l'époque des anciens réseaux qui faisaient confiance à la traction vapeur, ce qui semblait moins évident à la nouvelle SNCF. Mais Vilain, doué d'un véritable coup d'œil géographique, a immortalisé la vapeur sur les plus belles lignes de montagne, notamment dans le Massif central, et a été le seul auteur à entreprendre l'encyclopédie de la locomotive en France illustrée par ses clichés.

Les grands réseaux ont dû réagir face à la crise de l'entre-deuxguerres et montrer qu'ils se modernisaient. On ne pouvait pas les accuser de gaspiller de l'argent ni, surtout, de mauvaise gestion, et ils le faisaient savoir. Les ancêtres de La Vie du rail sont nés vers 1929 et avaient cette préoccupation. Le Bulletin du PLM travaillait avec l'agence Keystone et le Réseau de l'État, sous l'impulsion de Raoul Dautry, a demandé à François Kollar de réaliser un livre dans la collection « La France travaille » avec un texte de Pierre Hamp, ancien cheminot du Nord (tout comme Dautry) devenu inspecteur du travail. Les clichés sont aujourd'hui conservés par la Bibliothèque Forney et les plus beaux ont été publiés dans un ouvrage édité par La Vie du rail ${ }^{1}$.

1- Les Hommes de l'État: cheminots des années 30, photographies de François Kollar, Paris, La Vie du rail, 1986, 111 p. 
D’autres revues ferroviaires ont aussi publié des photographies de cheminots, mais plus rarement. Il faut dire aussi que parmi les grands noms de la photographie ferroviaire, Floquet, Broncard et Fénino, seul ce dernier a adopté une approche humaine, les premiers s'étant spécialisés dans les trains en marche et dans le paysage (fig. 1).

Peu d'amateurs ferroviaires ont vraiment photographié des cheminots au travail, à part Jean Florin et Maurice Maillet; bien que n'appartenant pas professionnellement à la SNCF, ils en étaient cependant très proches et n'hésitaient pas à monter à bord des locomotives à vapeur à l'époque où celles-ci circulaient dans l'indifférence générale ; le public manifestait alors un certain dédain pour ces machines noires et salissantes ; nous étions loin des trains de plaisir touristiques d'aujourd'hui !

Christian Schnabel s'est consacré aux lignes secondaires, notamment celles de la région parisienne dans les années 1950-1960 et on lui doit de belles photos d'ambiance ainsi que quelques gestes du métier repris dans un ouvrage de la collection «Images de trains » éditée par La Vie du rail ${ }^{2}$.

Travaillant au service photographique de cette dernière entreprise, ce n'est pas sans une certaine émotion que je retrouve parfois des séries complètes de reportages, sous forme de clichés, sur tel ou tel métier aujourd'hui disparu. Heureusement que les photographes de l'époque qui travaillaient pour La Vie du rail ont songé au patrimoine ferroviaire et humain (mais en avaient ils seulement conscience ?). En revanche ils devaient prendre un maximum de clichés, dont les plus significatifs paraissaient dans la rubrique : "Qu'avez vous fait de votre journée M. X (ou Madame)» où un(e) cheminot(e) racontait par l'image et le texte une plage de son activité, sans néanmoins indiquer son salaire (fig. 2). La Vie du rail, journal des cheminots, fait à l'époque par des cheminots journalistes, a bien pénétré le monde des travailleurs ferroviaires.

Et les syndicats ? On trouve bien chez eux quelques photos mais sans plus ; beaucoup de dessins dans La Vie ouvrière à l'époque de la nationalisation. En fait, le cheminot fait son travail sans gloriole et refuse de se faire photographier. Je me souviens des regards amusés des mécaniciens du dépôt des Joncherolles ce 12 décembre 1970, dernier jour de la traction vapeur en région parisienne. Certes ils regrettaient de perdre leur machine mais, pour eux, c'était une étape professionnelle de plus, et ils étaient un peu étonnés de nous voir les photographier avec leur locomotive et avoir plus de regrets qu'eux-mêmes (fig. 3).

2- Les Secondaires des années 50 et 60, avec les plus belles photos en couleur de Christian Schnabel, coll. «Images de trains », tome 7, Paris, La Vie du rail, 2000. 

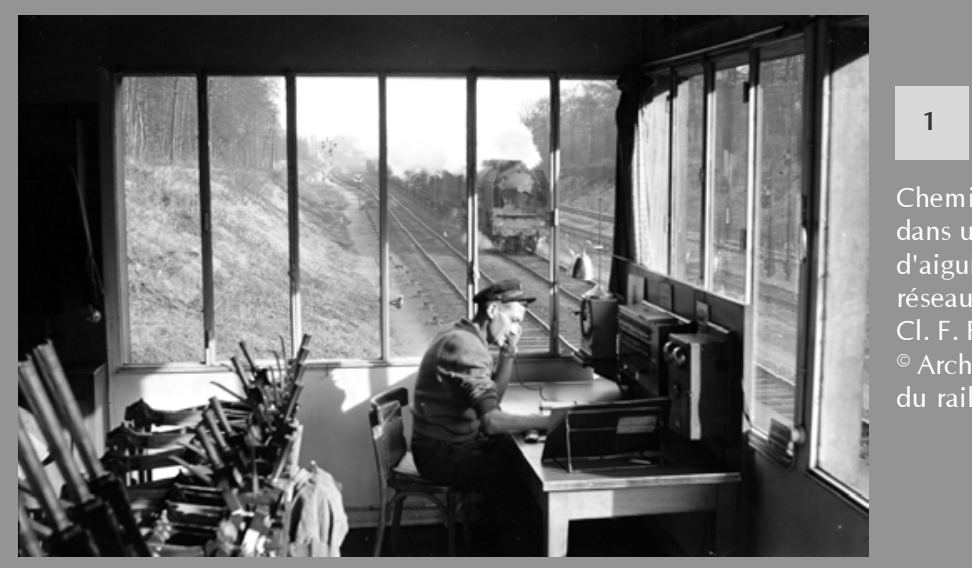

Cheminot au travail dans un poste d'aiguillage sur le réseau de l'Est. Cl. F. Fenino. ๑) Archives La Vie du rail.

2

Vie d'un facteur mixte dans une petite gare de province.

- Archives La Vie du rail.
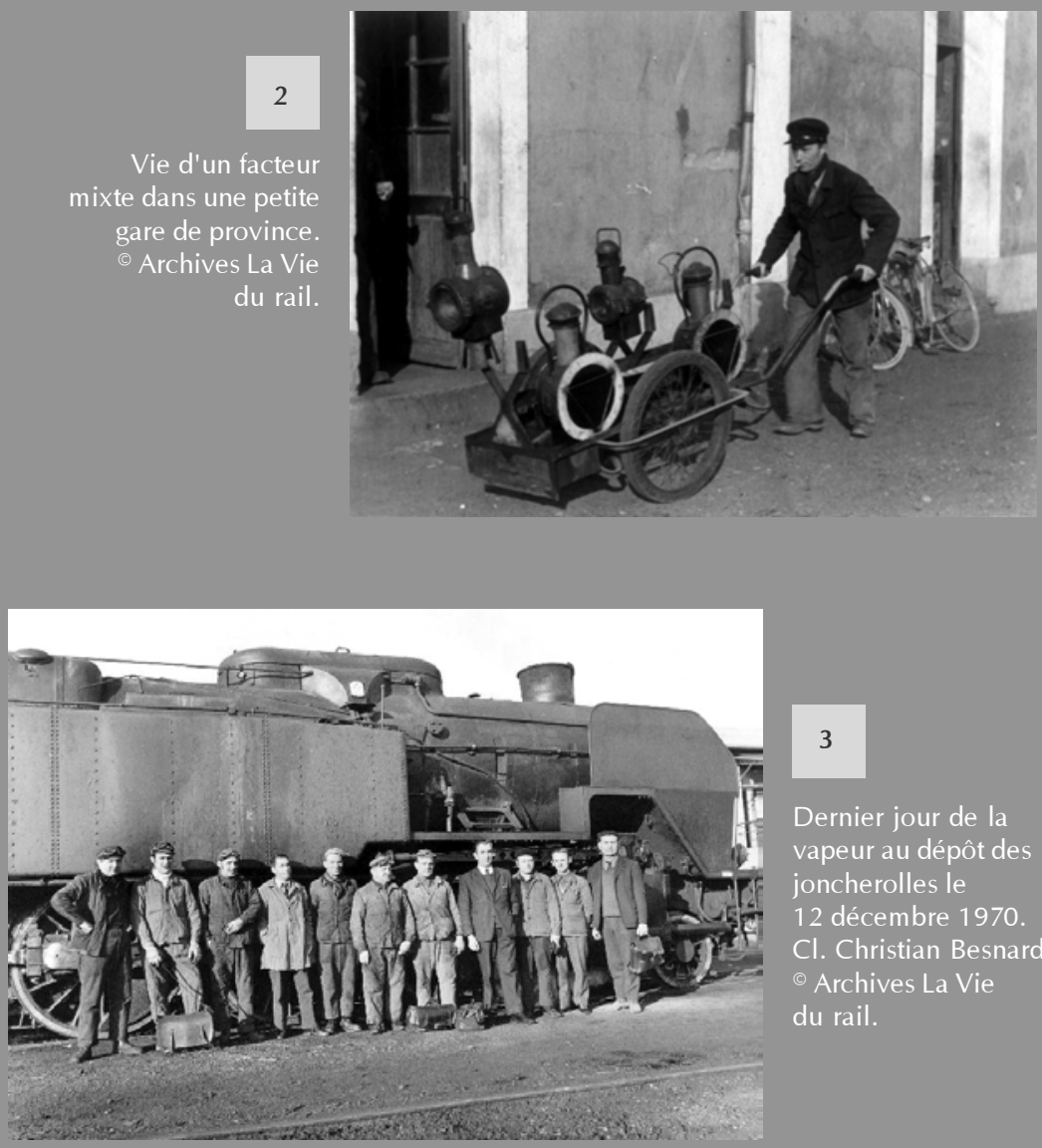

3

Dernier jour de la vapeur au dépôt des joncherolles le 12 décembre 1970. Cl. Christian Besnard. ( ) Archives La Vie du rail. 
Pour terminer, parlons des dessins et des photographies relatives à la sécurité du personnel. Au temps des anciens réseaux quelques photos existaient, notamment au Nord. La SNCF a repris le règlement PS 9A en dessins fidèles, montrant à la fois tout ce qu'il ne fallait pas faire (en rouge) et en bleu le geste à accomplir. Vers 1970, une version photographique apparaît et, à la même époque, La Vie du rail demande au dessinateur Arfoll (mon père) d'illustrer le «calepin Sécurité » avec deux personnages, Pépin et Parfait, en suivant les principes précédents.

Pour conclure, on trouve dans l'ouvrage de Georges Ribeill La Révolution ferroviaire ${ }^{3}$ quelques photos du xix ${ }^{e}$ siècle dont l'une, prise en 1869 à Saint-Lazare, a une allure très moderne, tout en sachant que parmi les participants à cette prise de vue certains ont pu naitre à la fin des années $1700 . .$. Ceci prouve bien que le chemin de fer a été bien conçu et bien perçu par l'ensemble des populations et que les gens qui le servent n'ont pas à rougir de leur travail même si l'iconographie sur le personnel est moins abondante que dans d'autres corps de métier.

3- La Révolution ferroviaire. La formation des compagnies de chemin de fer en France (18231870), préface de Jacques Fournier, Paris, Belin, 1993, 478 p. 